\title{
Zur Einschätzung der Minderung der Erwerbsfähigkeit infolge einer IgE-vermittelten Allergie mit Organmanifestation an Haut und Atemwegen
}

\author{
D. Nowak \\ T. L. Diepgen \\ H. Drexler
}

Konsenspapier der Deutschen Gesellschaft für Pneumologie, der Arbeitsgemeinschaft Berufsdermatologie in der Deutschen Dermatologischen

Gesellschaft und der Deutschen Gesellschaft für Arbeitsmedizin und Umweltmedizin e. $V$.
Reduced earning capacity due to IgE-mediated skin and airway allergy.
Consensus paper

\section{Einleitung}

In der unfallversicherungsrechtlichen Zusammenhangsbegutachtung ist es Aufgabe des medizinischen Sachverständigen, den Vollbeweis für das medizinische Krankheitsbild zu sichern und sich zur haftungsausfüllenden Kausalität zu äußern. Darüber hinaus ist regelmäßig eine Einschätzung der Minderung der Erwerbsfähigkeit (MdE) gefordert.

Als Pneumologe orientiert man sich an einschlägigen Empfehlungen, die vorrangig das Ausmaß der Funktionsbeeinträchtigung widerspiegeln. Streng genommen ist die MdE durch die Einschränkungen bedingt, sich auf dem allgemeinen Arbeitsmarkt einen Erwerb zu verschaffen. Neben der vom Pneumologen relativ leicht beurteilbaren funktionellen Einschränkung beinhaltet der MdE-Begriff somit eine Aussage über den allgemeinen Arbeitsmarkt. Hierfür sind wir eigentlich nicht ausreichend sachverständig, dennoch wird von uns eine Antwort verlangt.

Bei den berufsbedingten allergischen Erkrankungen besteht neben dem genannten - grundsätzlichen - Problem jeder unfallversicherungsrechtlichen MdE-Beurteilung ein weiterer Bruch in der Systematik zwischen zwei verschiedenen Zielorganen: Allergische Berufskrankheiten der Lunge werden gutachterlich nach systematisch anders aufgebauten Kriterien beurteilt als allergische Berufskrankheiten der Haut: Während in die einschlägigen MdE-Empfehlungen für allergische obstruktive Atemwegserkrankungen nur das Ausmaß der Organschädigung eingeht, benutzen die Dermatologen seit Jahren zwei Kriterien: Neben dem Ausmaß der Organschädigung wird die Auswirkung der Allergie auf dem allgemeinen Arbeitsmarkt berücksichtigt. Faktisch bedeutet dies, dass wir einem Patienten mit einem abgeklungenen Latex-Asthma, CAP-Klasse 2 auf Latex, normaler Lungenfunktion und vielleicht noch diskret erhöhter unspezifischer Atemwegsempfindlichkeit in der Regel eine MdE von unter 20 v.H. (0 oder meist 10 v.H.) zuerkennen würden. Die Dermatologen hingegen würden bei einem Patienten mit einer abgeklungenen Latex-Dermatitis, CAP-Klasse 2 und erscheinungsfreier Haut durchaus eine MdE von 20 v.H. konstatieren. Das bedeutet in praxi, dass die abgeklungene Dermatitis vielfach mit einer Rentenzahlung einhergeht, das abgeklungene Asthma jedoch nicht.

Bei vordergründiger Betrachtung erscheint die dermatologische Sichtweise, welche die Auswirkung der Allergie auf dem allgemeinen Arbeitsmarkt berücksichtigt, der Legaldefinition der MdE besser zu entsprechen als die pneumologische Betrachtungsweise, welche praktisch allein den Funktionsschaden beschreibt. Allerdings besteht das Kernproblem der dermatologischen Empfehlungen darin, dass keine Daten zur Verbreitung von Typ I-Allergenen auf dem allgemeinen Arbeitsmarkt vorliegen, somit die Auswirkung der Allergie gar nicht quantifiziert werden kann. Für einige Typ IV-Allergien liegen entsprechende Daten vor, nicht jedoch für Typ I-Allergene.

Es ist das Anliegen des nachfolgend abgedruckten Konsenspapiers, auf diese Problematik aufmerksam zu machen und wis- 
senschaftliche Untersuchungen zu fordern, die eine fundiertere MdE-Einschätzung erlauben. Letztlich soll es dazu dienen, die Begutachtung auch organübergreifend gerechter zu gestalten.

\section{Konsensuspapier}

Ausgelöst durch die hohe Zahl von Personen mit einer Berufskrankheit infolge einer Latex-Allergie wurde mehrfach auf die diskrepanten Empfehlungen der Deutschen Gesellschaft für Pneumologie (Kroidl, Nowak, Seysen, Bewertung und Begutachtung in der Pneumologie, 2. Auflage 2000) und der Arbeitsgemeinschaft für Berufsdermatologie (Diepgen, Kühl, Schmidt, Dermatosen 43, 290-292, 1995) hingewiesen. Ein Konsensgespräch zwischen von den Präsidenten der genannten Fachgesellschaften beauftragten Vertretern fand in Heidelberg statt (für die DGP: D. Nowak, München; für die AG Berufsdermatologie in der DDG: T. L. Diepgen, Heidelberg; für die DGAUM: H. Drexler, Erlangen). Über folgende Punkte wurde Einigkeit erzielt.

- Die zunehmende Zahl Latex-allergischer Patienten war der Auslöser, der die Diskrepanzen zwischen pneumologischer und dermatologischer MdE-Einschätzung aufzeigte, jedoch ist die Latex-Allergie nicht die einzige berufsbedingte Тур I-Allergie, die mit Symptomen an verschiedenen Organsystemen einhergehen kann.

- Die gutachterliche Bewertung der Sensibilisierung und der dadurch verschlossenen Bereiche des allgemeinen Erwerbslebens sollte zwischen den Fachgesellschaften aus Gründen der Gleichbehandlung der Versicherten nach analogen Prinzipien erfolgen, was bislang nicht der Fall ist.

- Die wesentliche Diskrepanz zwischen der MdE-Einschätzung auf pneumologischem und dermatologischem Fachgebiet besteht darin, dass auf pneumologischem Fachgebiet (BK 4301) bei bestehender Sensibilisierung ohne funktionelle Beeinträchtigungen an den Atemwegen (normale Lungenfunktion, regelrechte oder asymptomatisch gesteigerte Atemwegsempfindlichkeit) eine MdE stets unter $20 \mathrm{v}$. H. beigemessen wird, während auf dermatologischem Fachgebiet (BK 5101) bei Vorliegen einer Sensibilisierung ohne aktuelle Krankheitserscheinungen und ohne Funktionseinschränkungen eine MdE bis 20 v. H. resultieren kann.

- Maßgeblich für die Bewertung der Erwerbsfähigkeit ist die durch die Berufskrankheit bedingte Einschränkung, sich auf dem allgemeinen Arbeitsmarkt einen Erwerb zu verschaffen. Die Bewertung der Auswirkungen einer Allergie umfasst dabei neben den Auswirkungen der Erkrankung den Anteil der durch die Allergie verschlossenen Arbeitsplätze. Der Grad der Sensibilisierung, das Ausmaß der klinischen Erscheinungen und die Verbreitung des Allergens gehen in die MdE-Bemessung ein. Bezüglich der Verbreitung des Allergens ist dessen Vorkommen in krankheitsauslösender bzw. -unterhaltender Form zugrunde zu legen. Es bleibt zu prüfen, ob es überhaupt Typ I-Allergene (einschließlich kreuzreagierender Allergene) gibt, die auf dem allgemeinen Arbeitsmarkt in krankheitsauslösender bzw. krankheitsunterhaltender Konzentration so weit verbreitet sind, dass dadurch $20 \%$ der Arbeitsplätze verschlossen sind. Eine gesicherte Datenbasis, auf die zurückgegriffen werden könnte, ist nicht vorhanden. Bei Erscheinungsfreiheit im Bereich des Hautorgans ist eine MdE von $20 \mathrm{v}$.H. folglich nicht begründbar.

- Wenn infolge einer allergischen Erkrankung eine Berufskrankheit nach BK 4301 und 5101 BKV anzuerkennen ist, kann aus medizinischen Gründen in der Regel die Addition der MdE-Sätze nicht sinnvoll sein, da meist dieselben Arbeitsplätze verschlossen sind. Aus medizinischer Sicht ist in diesem Fall die Einschätzung einer Gesamt-MdE sachgerechter. 